\title{
ON KRULL OVERRINGS OF A NOETHERIAN DOMAIN
}

\author{
WII.LIAM HEINZER
}

An integral domain $D$ is called a Krull ring if $D=\bigcap_{\alpha} V_{\alpha}$ where $\left\{V_{\alpha}\right\}$ is a set of rank one discrete valuation rings with the property that for each nonzero $x$ in $D, x V_{\alpha}=V_{\alpha}$ for all but a finite number of the $V_{\alpha}$. If $D$ is a Krull ring and $\left\{P_{\alpha}\right\}$ is the set of primes of height one (minimal primes) of $D$, then each localization $D_{P_{\alpha}}$ is a rank one discrete valuation ring and $D=\bigcap_{\alpha} D_{P_{\alpha}}$ is an irredundant representation of $D$. The set $\left\{D_{P_{\alpha}}\right\}$ is called the set of essential valuation rings for $D$. We use the notation $E(D)$ to denote the set of essential valuation rings of a Krull ring $D$. Important examples of Krull rings are noetherian integrally closed domains and unique factorization domains. The one dimensional Krull rings are precisely the Dedekind domains. Hence one dimensional Krull rings are noetherian. An example of a three dimensional nonnoetherian Krull ring is provided by Nagata's example [6, p. 207] of a three dimensional noetherian domain whose derived normal ring is not noetherian; for it is known that the derived normal ring of a noetherian domain is Krull [6, p. 118]. Our purpose here is to show that a rather extensive class of two dimensional Krull rings are noetherian. ${ }^{1}$ If $R$ is an integral domain with quotient field $K$ we call a domain $D$ such that $R \subseteq D \subseteq K$ an overring of $R$. We show that if $R$ is a two dimensional noetherian domain, then each Krull overring of $R$ is noetherian. Since the derived normal ring of a two dimensional noetherian domain is again noetherian $[6$, p. 120], our problem is reduced to showing that a Krull overring of a two dimensional noetherian Krull ring is noetherian. Important arguments used in the proof were obtained from [8].

LemMa 1. Let $R$ be a noetherian domain and let $P$ be a prime ideal of $R$ having depth $\leqq 1$. Suppose that $D$ is an overring of $R$ having a prime ideal $Q$ such $Q \cap R=P$. If $D \subseteq R_{P}$, then $D / Q$ is noetherian.

Proof. We have $R / P$ canonically imbedded in $D / Q$ and the fact that $D \subseteq R_{P}$ implies that $D / Q$ is contained in the quotient field of $R_{\prime}^{\prime} P$. Since $R / P$ is a noetherian domain of dimension $\leqq 1$, it follows by the Krull-Akizuki Theorem that $D / Q$ is also noetherian [3].

COROLlaRy 2. Let $R$ be a two dimensional noetherian domain and let

Received by the editors November 11, 1968.

${ }^{1}$ That not every two dimensional Krull ring is noetherian is shown by an example in [4]. 
$P$ be a prime ideal of $R$ of height one such that $R_{P}$ is integrally closed. If $D$ is an overring of $R$ and $Q$ is a prime ideal of $D$ such that $Q \cap R=P$, then $D / Q$ is noetherian.

Proof. We have $R_{P} \subseteq D_{Q}$; and $R_{P}$ integrally closed implies that $R_{P}$ is a discrete rank one valuation ring, so that $R_{P}=D_{Q}$. Thus $D \subseteq R_{P}$ and the corollary follows from Lemma 1.

We recall that if $J$ is a commutative ring and $X$ is an indeterminate over $J$, then $J(X)$ denotes the ring of quotients $J[X]_{S}$ where $S$ is the multiplicative system of $J[X]$ consisting of all polynomials $f$ in $J[X]$ such that the coefficients of $f$ generate the unit ideal of $J[6$, p. 18]. We will have occasion to use the following known facts about $J(X)$.

(i) If $J$ is noetherian, then $J(X)$ is noetherian and $\operatorname{dim} J$ $=\operatorname{dim} J(X)$.

(ii) If $J$ is Krull, then $J(X)$ is Krull; and if $K$ is the quotient field of $J$, then the set of essential valuation rings of $J(X)$ is precisely the set of essential valuation rings for $K[X]$ that contain $J(X)$ together with the canonical extensions to $K(X)$ of the essential valuation rings for $J$.

(iii) If $J$ is Krull and $Q$ is an ideal of $J$ contained in no height one prime of $J$, then $Q J(X)$ contains a nonzero principal prime of $J(X)$. In fact, if $a, b \in Q$ are chosen so that $(a, b)$ is contained in no height one prime of $J$, then $a X+b$ is prime in $J(X)$. Note that $(a X+b)$ is the center on $J(X)$ of an essential valuation ring of $K[X], K$ the quotient field of $J$.

(iv) $J(X)$ is faithfully flat over $J$. Thus each ideal of $J$ extends and contracts to itself with respect to $J(X)[2$, p. 50]; and if an ideal $Q$ of $J$ is such that $Q J(X)$ is finitely generated, then $Q$ is also finitely generated.

Theorem 3. Let $R$ be a two dimensional noetherian integrally closed domain and let $D$ be a Krull overring of $R$. If $Q$ is an ideal of $D$ contained in no height one prime of $D$, then $Q$ is finitely generated.

Proof. Let $K$ be the quotient field of $R$ and let $X$ be an indeterminate over $K$. We have $R(X) \subseteq D(X)$ and by (iii) there exists a principal prime of $D(X)$, say $P_{1}$, such that $P_{1} \subseteq Q D(X)$ and such that $K[X] \subseteq D(X)_{P_{1}}$. Let $P=P_{1} \cap R(X)$. Since $D(X)_{P_{1}}$ is an essential valuation ring for $K[X]$, we see from (ii) that $R(X)_{P}=D(X)_{P_{1}}$ and $P$ is a height one prime of $R(X)$. By Corollary $2, D(X) / P_{1}$ is noetherian. Since $P_{1}$ is finitely generated, we see that $Q D(X)$ and hence $Q$ is finitely generated. 
Lemma $4 .^{2}$ Let $J$ be a Krull ring and let $V$ be an essential valuation ring for $J$ whose center $N$ on $J$ is a maximal ideal. Let $J^{\prime}$ be the Krull overring of $J$ having $E(J)-\{V\}$ as its set of essential valuation rings. If $P^{\prime}$ is a finitely generated prime ideal of $J^{\prime}$ then $P^{\prime} \cap J=P$ is also finitely generated. If $J^{\prime}$ is noetherian it follows that $J$ is noetherian.

Proof. We note that $J^{\prime}$ is the $N$-transform of $J$ as defined by Nagata in $\left[7\right.$, p. 58]. Also $N J^{\prime} \cap J$ properly contains $N$ so that $N J^{\prime}=J^{\prime}$. Hence there is a one to one correspondence between the prime ideals of $J^{\prime}$ and the prime ideals of $J$ excluding $N$ where a prime ideal $P^{\prime}$ of $J^{\prime}$ is associated with $P^{\prime} \cap J=P$ and $J_{p^{\prime}}^{\prime}=J_{P}[7$, p. 58] or $\left[8\right.$, p. 198]. We choose $\left\{x_{1}, \cdots, x_{n}\right\}=X \subseteq N$ so that $X J^{\prime}=J^{\prime}$. We may also assume that $X J_{N}=N J_{N}$. Then $X J=N$ for if $P$ is a maximal ideal of $J$ distinct from $N$ we have $X J_{P}=N J_{P}=J_{P}$. Thus $N$ is finitely generated. ${ }^{3}$ If $P^{\prime}$ is a finitely generated prime of $J^{\prime}$ with $P$ $=P^{\prime} \cap J$ we choose $\left\{y_{1}, \cdots, y_{m}\right\}=Y$ in $J^{\prime}$ so that $Y J^{\prime}=P^{\prime}$. There exists an integer $t$ such that $Y N^{t} \subseteq J$. Hence $Y J \cdot N^{t}=B$ is a finitely generated ideal of $J$ such that $B J^{\prime}=P^{\prime}$. By enlarging $B$ if necessary we may assume that $B \nsubseteq N$. Thus $B J_{N}=P J_{N}=J_{N}$. If $M$ is a maximal ideal of $J$ distinct from $N$ and $M^{\prime}$ is the unique maximal ideal of $J^{\prime}$ with $M^{\prime} \cap J=M$, then $B J_{M^{\prime}}^{\prime}=P^{\prime} J_{M^{\prime}}=P J_{M}$. It follows that $B=P$ $\left[10\right.$, p. 94]. Thus $P^{\prime}$ finitely generated implies that $P$ is finitely generated. Since $N$ is the only prime of $J$ that is not the contraction of a prime of $J^{\prime}$ we see that $J^{\prime}$ noetherian implies that $J$ is noetherian.

THEOREM 5. Let $R$ be a two dimensional noetherian integrally closed domain and let $D$ be a Krull overring of $R$. If $P_{1}$ is a prime ideal of $D$ such that $P_{1} \cap R=P$ is of height one, then $P_{1}$ has a finite basis.

Proof. We note that the finiteness condition in the definition of a Krull ring insures that only finitely many $V \in E(D)$ can be such that the center of $V$ on $R$ contains $P$. If $V$ has center $M$ on $R$ where $M$ is a prime of height two, then by the $R$-dimension of $V$ we will mean the transcendence degree over $R / M$ of the residue field of $V$. (Here we are using the canonical embedding of $R / M$ in the residue field of $V$.) Since $R$ is two dimensional and noetherian each such $V$ has $R$-dimension either zero or one $[1$, p. 328]. Moreover, if $V$ has $R$-dimension

2 This lemma also appears in [5] where it is shown that a certain class of two dimensional normal affine rings have the property that each Krull overring is noetherian.

${ }^{3}$ We have in fact shown that a height one prime of a Krull ring which is also maximal is invertible, for $N$ is finitely generated and localized at any maximal ideal $N$ is principal. 
zero, then $V$ is necessarily centered on a maximal ideal of any domain between $R$ and $V$. Let $\left\{V_{i}\right\}$ be the subset of $E(D)$ consisting of all $V \in E(D)$ such that the center of $V$ on $R$ properly contains $P$ and such that the $R$-dimension of $V$ is zero. Let $D^{\prime}$ be the Krull overring of $D$ having $E(D)-\left\{V_{i}\right\}$ as its set of essential valuation rings. We note that $R_{P}=D_{P_{1}}$ is in the set $E\left(D^{\prime}\right)$ and Lemma 4 implies that if the center of $R_{P}$ on $D^{\prime}$ is finitely generated then $P_{1}$ the center of $R_{P}$ on $D$ is also finitely generated. Let $\left\{W_{i}\right\}$ be the subset of $E\left(D^{\prime}\right)$ consisting of all $W \in E\left(D^{\prime}\right)$ such that the center of $W$ on $R$ properly contains $P$. We note that $\left\{W_{i}\right\}$ is a finite set and that each $W_{i}$ has $R$-dimension one. Let $T_{i}$ be the maximal ideal of $W_{i}$. Since $W_{i}$ is a quotient ring of $D^{\prime}$ we see that $D^{\prime} / T_{i} \cap D^{\prime}$ has quotient field $W_{i} / T_{i}$. We choose $a_{i}$ in $D^{\prime}$ such that the residue of $a_{i}$ in $W_{i} / T_{i}$ is transcendental over $R / T_{i}$ $\cap R$. Then $W_{i}$ is not centered on a maximal ideal of $R\left[a_{i}\right]$ so that $W_{i}$ is necessarily an essential valuation ring for $R^{\prime}$, the integral closure of $R\left[a_{1}, \cdots, a_{n}\right]$. Since $R^{\prime}$ is the derived normal ring of a noetherian domain of dimension $\leqq 2, R^{\prime}$ is noetherian. Let $P^{\prime}$ denote the center of $R_{P}$ on $R^{\prime}$ and let $P_{1}^{\prime}$ denote the center of $R_{P}$ on $D^{\prime}$. We have $P_{1}^{\prime} \cap R^{\prime}=P^{\prime}$ and $P^{\prime}$ is not contained in the center of any $V \in E\left(D^{\prime}\right)$ distinct from $R_{P}$. It remains to prove that $P_{1}^{\prime}$ is finitely generated. We use an argument from $\left[8\right.$, p. 204]. Let $Q=P^{\prime} D^{\prime}: P_{1}^{\prime}$ $=\left\{d \in D^{\prime}: d P_{1}^{\prime} \subseteq P^{\prime} D^{\prime}\right\}$. We note that $Q$ is contained in no height one prime of $D^{\prime}$. Since $P^{\prime} \subseteq Q$ it is clear that $Q$ is contained in no height one prime of $D^{\prime}$ distinct from $P_{1}^{\prime}$. Because $R^{\prime}$ is a Krull ring there exists $x \in\left(P^{\prime}\right)^{-1}-R^{\prime}$. Since $P_{1}^{\prime} \cap R^{\prime}=P^{\prime}$ we can modify $x$ if necessary so that $x \in\left(P_{1}^{\prime}\right)^{-1}$ and $x \in\left(P^{\prime}\right)^{-1}-R^{\prime}$. Let $y \in P^{\prime}$ be such that $y x \in R^{\prime}-P^{\prime}$. Then $y x P_{1}^{\prime} \subseteq P^{\prime} D^{\prime}$ so that $y x \in Q$ and $Q$ is not contained in $P_{1}^{\prime}$. Theorem 3 now implies that $Q$ is finitely generated. Hence $Q / P_{1}^{\prime} Q$ is a finite $D^{\prime} / P_{1}^{\prime}$-module. By Corollary $2, D^{\prime} / P_{1}^{\prime}$ is noetherian so that $Q \cap P_{1}^{\prime} / P_{1}^{\prime} Q$ is also a finite $D^{\prime} / P_{1}^{\prime}$-module. Since $P^{\prime} D^{\prime}$ has a finite basis and $Q P_{1}^{\prime} \subseteq P^{\prime} D^{\prime}$ we see that $Q \cap P_{1}^{\prime}$ is finitely generated. By Theorem 3, each ideal containing $Q$ is finitely generated so that $D^{\prime} / Q$ is noetherian. Moreover, $D^{\prime} / Q$ and $D^{\prime} / P_{1}^{\prime}$ being noetherian implies that $D^{\prime} / Q \cap P_{1}^{\prime}$ is noetherian $[6$, p. 11]. Thus $P_{1}^{\prime} / Q \cap P_{1}^{\prime}$ is finitely generated. Since $Q \cap P_{1}^{\prime}$ has a finite basis we see that $P_{1}^{\prime}$ does also. This completes the proof of Theorem 5.

COROLlaRy 6. Let $R$ be a two dimensional noetherian integrally closed domain. If $D$ is a Krull overring of $R$ such that $E(D) \subseteq E(R)$, then $D$ is noetherian.

Proof. Since $E(D) \subseteq E(R)$ each prime of height one of $D$ contracts 
to a height one prime of $R$. Hence by Theorem 5, the primes of height one of $D$ are finitely generated. Theorem 3 implies that every prime of $D$ of height $>1$ is finitely generated so $D$ is noetherian.

COROLlaRY $7 .{ }^{4}$ If $R$ is a two dimensional noetherian integrally closed domain and $A$ is an ideal of $R$, then the $A$-transform $T$ of $R$ is again noetherian.

Proof. It is shown in $[8$, p. 198] that $T$ is a Krull overring of $R$ and that $E(T) \subseteq E(R)$.

Lemma 8. Let $R$ be a two dimensional normal local ring with maximal ideal $M$. If $D$ is a Krull overring of $R$, then $D$ is noetherian.

Proof. We note that $E(D)-E(R)$ is the finite set consisting precisely of the $V \in E(D)$ having center $M$ on $R$. Let $D^{\prime}$ be the Krull overring of $D$ such that $E\left(D^{\prime}\right) \subseteq E(D)$ and $E(D)-E\left(D^{\prime}\right)$ is precisely the $V \in E(D)-E(R)$ that have $R$-dimension zero. Since each such $V$ is centered on a maximal ideal of $D$, Lemma 4 implies that $D$ is noetherian if $D^{\prime}$ is noetherian. As in the proof of Theorem 5 we see easily the existence of a finite extension of $R$ whose derived normal ring $R^{\prime}$ is such that $E\left(D^{\prime}\right) \subseteq E\left(R^{\prime}\right)$. Hence by Corollary $6, D^{\prime}$ is noetherian.

THEOREM 9. If $D$ is a Krull overring of a two dimensional noetherian domain $R$, then $D$ is noetherian.

Proof. Since the derived normal ring of $R$ is noetherian and contained in $D$ we may assume that $R$ is integrally closed. Let $P_{1}$ be a prime ideal of $D$ and let $P=P_{1} \cap R$. If $P$ has height one, then Theorem 5 implies that $P_{1}$ is finitely generated. If $P$ has height two and $S$ $=R-P$, then $R_{S} \subseteq D_{S}$ so by Lemma $8, D_{S}$ is noetherian. Let $X_{1}$ be a finite subset of $P_{1}$ such that $X_{1} D_{S}=P_{1} D_{S}$ and let $X_{2}$ be a finite set of generators for $P$. We see that $X=X_{1} \cup X_{2}$ generates $P_{1}$. For if $M$ is a maximal ideal of $D$ and $M \cap R \neq P$, then $P_{1} D_{M}=D_{M}=X D_{M}$. If $M \cap R=P$, then $D_{S} \subseteq D_{M}$ so that $X D_{M}=P_{1} D_{M}$. Thus each prime of $D$ has a finite basis and $D$ is noetherian.

\section{REFERENCES}

1. S. Abhyankar, On the valuations centered on a local domain, Amer. J. Math. 78 (1956), 321-348.

\footnotetext{
4 An $A$-transform of a three dimensional noetherian domain need not be noetherian. In [4] it is shown that Rees' example [9] of an $A$-transform of a three dimensional normal affine ring that is not an affine ring is also not noetherian.
} 
2. N. Bourbaki, Éléments de mathématiques. Algèbre commutative, Chapitre 1, Hermann, Paris, 1961.

3. I. Cohen, Commutative rings with restricted minimum condition, Duke Math. J. 17 (1950), 27-42.

4. P. Eakin and W. Heinzer, Non-finiteness in finite dimensional Krull domains, J. Algebra (to appear).

5. W. Heinzer, On Krull overrings of an affine ring, Pacific J. Math. (to appear).

6. M. Nagata, Local rings, Interscience, New York, 1962.

7. —_, A treatise on the 14th problem of Hilbert, Mem. Coll. Sci. Univ. Kyoto Ser. A Math. 30 (1956-1957), 57-70.

8. - A theorem on finite generation of a ring, Nagoya Math. J. 27 (1966), 193-205.

9. D. Rees, On a problem of Zariski, Illinois J. Math. 2 (1958), 145-149.

10. O. Zariski and P. Samuel, Commutative algebra, Vol. II, Van Nostrand, Princeton, N. J., 1960.

Louisiana State University aNd

Purdue University 